https://doi.org/10.1007/s00350-018-4936-6

\section{Anmerkung zu BGH, Beschl. v. 26.9.2017 - VI ZR 81/17 (OLG München)}

\section{Martin Zurlinden}

I. Die Entscheidung betrifft zwei Gehörsrügen, die vom $\mathrm{BGH}$ in Anwendung des allgemeinen Grundsatzes der Subsidiarität unterschiedlich behandelt werden:

Zum einen hatte das Berufungsgericht in einem Hinweisbeschluss gemäß $\ 522$ Abs. 2 S. 2 ZPO darauf hingewiesen, dass der klägerische Vorwurf der fehlenden Operationsindikation mit der Berufung nicht weiterverfolgt werde. Dem BGH zufolge war dieser Hinweis verfehlt, da der Kläger in der Berufungsbegründung deutlich gemacht habe, dass er auch die Bejahung der Indikation durch das Erstgericht angreifen wolle. Der Kläger habe es jedoch trotz eingeräumter Stellungnahmefrist versäumt, das Berufungsgericht auf den Gehörsverstoß aufmerksam zu machen, weshalb er im Nichtzulassungsbeschwerdeverfahren mit der Rüge ausgeschlossen sei.

Anders beurteilt der BGH die Rüge eines weiteren Gehörsverstoßes. Das Berufungsgericht habe einen Widerspruch zwischen den Ausführungen des Privatgutachters und denen des gerichtlichen Sachverständigen verneint, obwohl der Widerspruch eindeutig gewesen sei, was den Schluss zulasse, dass das Berufungsgericht den Akteninhalt unter Verstoß gegen Art. 103 Abs. 1 GG nur selektiv zur Kenntnis genommen habe. Mit der entsprechenden Rüge sei der Kläger nicht ausgeschlossen, obwohl er dem Hinweis des Berufungsgerichts, ein Widerspruch sei nicht zu erkennen, nicht entgegengetreten sei. Zur Begründung führt der $\mathrm{BGH}$ aus, dass das Berufungsgericht seine (unzutreffende) Beurteilung nicht begründet habe und der Kläger nicht gehalten gewesen sei, seinen bereits in der Berufungsbegründung enthaltenen Vortrag zur Widersprüchlichkeit der Gutachten zu wiederholen.

Die vom BGH vorgenommene Differenzierung überzeugt (zweifelnd Toussaint, FD-ZVR 2017, 398754). Der erste Gehörsverstoß lag aufgrund des Hinweisbeschlusses für den Kläger klar auf der Hand. Hingegen drängte sich der zweite Gehörsverstoß nicht auf, denn immerhin hatte das Berufungsgericht im Hinweisbeschluss zu erkennen gegeben, dass es den klägerischen Vorwurf der Widersprüchlichkeit der Gutachten zur Kenntnis genommen hatte. Den Parteien bzw. Parteivertretern kann indes nur geraten werden, jeden Anhaltspunkt für einen Gehörsverstoß zum Gegenstand einer rechtzeitigen Stellungnahme zu machen, um einen Rügeverlust nach dem Grundsatz der Subsidiarität zu vermeiden.

II. Der Beschluss gibt noch Anlass zu einigen Anmerkungen zum Berufungsrecht:

Der BGH führt unter näheren Darlegungen aus, der Kläger habe hinreichend klar zu erkennen gegeben, dass er den Vorwurf der fehlenden Operationsindikation mit der Berufung weiterverfolgen wolle. Daneben stand offenbar der Vorwurf, die Operation sei auch fehlerhaft durchgeführt worden. Die Ausführungen des BGH sind vor dem Hintergrund der vom Kläger mit der Nichtzulassungsbeschwerde erhobenen Gehörsrüge zu sehen. Sie dürfen nicht zu der Annahme verleiten, dass einzelne Behandlungsfehlervorwürfe, die mit der Berufung nicht wiederholt werden, im Berufungsverfahren ohne Weiteres außer Betracht bleiben dürften (oder gar müssten). Der Prüfungsumfang des Berufungsgerichts wird durch den Streitgegenstand des Berufungsverfahrens bestimmt. Grundsätzlich ist das gesamte Behandlungsgeschehen, soweit es zum Streitgegenstand gehört, zu überprüfen, ohne dass

Dr. iur. Martin Zurlinden,

ROLG, OLG Hamm, 3. Zivilsenat (Arzthaftung),

Heßlerstraße 53, 59065 Hamm, Deutschland der Berufungsführer jeden erstinstanzlichen Fehlervorwurf noch einmal aufgreifen müsste, auch nicht unter dem Aspekt des $₫ 520$ Abs. 3 S. 2 ZPO (vgl. BGH, VersR 2017, 822 Rdnrn. 16-22 = MedR 2017, 799; s. auch BGH, NJW 2015, 3040, Rdnrn. 9 ff. zu mehreren Prospektmängeln). Eine Beschränkung des Streitstoffs auf einzelne Elemente der streitgegenständlichen Behandlung ist möglich, dies setzt aber einen eindeutig erkennbaren Beschränkungswillen der fraglichen Partei voraus (vgl. BGH, NJW 2015, 3040, Rdnr. 9).

Freilich wird und darf sich das Berufungsgericht in der Regel auf die mit der Berufung vorgebrachten Fehlervorwürfe konzentrieren. (Nähere) Ausführungen zum übrigen streitgegenständlichen Behandlungsgeschehen sind entbehrlich, wenn dieses - unter Würdigung der erstinstanzlichen Beweisaufnahme - keinen Anhalt für Behandlungsfehler bietet. Dies gilt erst recht dann, wenn sich bereits das Erstgericht überzeugend mit dem übrigen Behandlungsgeschehen auseinandergesetzt hat.

$\mathrm{Zu}$ ergänzen ist noch Folgendes: Hat das Erstgericht eine auf Behandlungsfehlervorwürfe und Aufklärungsrügen gestützte Klage abgewiesen und befasst sich die Berufung allein mit der Aufklärungsrüge, dann sind mögliche Behandlungsfehler nicht Gegenstand der Berufung (und umgekehrt), vgl. BGH, MedR 2007, 722, Rdnrn. 9-12.

III. Fazit: Die Entscheidung des BGH zeigt erneut, dass etwaige gerichtliche Gehörsverstöße frühzeitig gerügt werden müssen. Rückschlüsse auf die richtige Gestaltung des erstund zweitinstanzlichen Verfahrens lässt der Beschluss nur eingeschränkt zu. Generell ist bei der Interpretation von Entscheidungen über Nichtzulassungsbeschwerden zu bedenken, dass sich die Prüfung durch den BGH auf die (vom Beschwerdeführer dargelegten) Zulassungsgründe des $₫ 543$ Abs. 2 S. 1 ZPO beschränkt. Die Zurückweisung einer Nichtzulassungsbeschwerde darf nicht ohne Weiteres als Bestätigung des Berufungsgerichts in der Sache verstanden werden.

\section{Keine Persönlichkeitsrechtsverletzung durch Live-Übertragung einer Operation zu Aus- und Fortbildungszwecken}

GG Art. 1 Abs. 1, 2 Abs. 2; BGB §§253 Abs. 2, 823 Abs. 1 und Abs. 2; KunstUrhG §§22, 23

1. Will die Arztseite unter der Operation (hier: während der Narkose) Fotos oder sonstige Aufnahmen vom Patienten anfertigen, so hat sie grundsätzlich zuvor dessen Einwilligung einzuholen.

2. Die Live-Übertragung einer seltenen Operation zum Zweck der Aus- und Fortbildung stellt jedenfalls dann keinen erheblichen Eingriff in das allgemeine Persönlichkeitsrecht des Patienten dar, wenn auf dem Bildschirm nur das unmittelbare, außerhalb des Schambereichs liegende Operationsgebiet (hier: ein Ausschnitt der rechten Gesichtshälfte) zu erkennen und jede Möglichkeit einer Identifizierung des Patienten ausgeschlossen ist.

OLG Bamberg, Hinweisbeschl. v. 30. 6. 2016 u. (End-)Beschl.

v. 11.8.2016- 4 U 103/16 (LG Würzburg)

Problemstellung: Das OLG Bamberg sah sich mit der Frage konfrontiert, ob die Live-Übertragung einer Operation zum Zwecke der Aus- und Fortbildung von

Eingesandt von VRiOLG Peter Herdegen, Bamberg;

bearbeitet von Anna Maria Ernst, LL.M.

Wissenschaftliche Mitarbeiterin am Institut für Medizinrecht, Universität Köln,

Albertus-Magnus-Platz, 50923 Köln, Deutschland 